\title{
Reliability, risk and safety: analytical support for decision making
}

\author{
Lesley Walls ${ }^{1} \cdot$ Tim Bedford ${ }^{1} \cdot$ Matthew Revie $^{1} \cdot$ Enrico Zio $^{2,3}$
}

Received: 26 September 2017/Accepted: 28 September 2017/Published online: 23 October 2017

(C) Springer-Verlag GmbH Germany and EURO - The Association of European Operational Research Societies 2017

\section{Introduction}

Ensuring safe and reliable engineering systems involves making risk-informed decisions typically in the presence of uncertainties. Modelling and analysis play an important role in the decision-making process. Useful modelling addresses important problems, is grounded in sound methodology and is cognisant of the needs of the decision-maker. Reliability, risk and safety are taken to include the coverage of issues such as, for example, availability, maintenance, risk and resilience, with application areas including energy, transportation, manufacturing, critical infrastructure, oil and gas, chemical, mechanical amongst others.

This Feature issue presents considered insights into the role of modelling and analysis to support the process of making decisions aimed at managing engineered products and industrial plant safely and reliably so that good choices of action can be made and compliance against specified criteria can be established. The articles have been developed from papers presented at the European Safety and Reliability Conference (ESREL2016) held at the University of Strathclyde, Glasgow, Scotland in September 2016. The ESREL annual conference series is the main activity of the European Safety and Reliability Association ESRA (www.esrahomepage.eu). It stands as one of the most relevant and recognized conferences on the topics of safety

Lesley Walls

lesley.walls@strath.ac.uk

1 University of Strathclyde, Glasgow, Scotland, UK

2 CentraleSupélec, Gif sur Yvette, France

3 Politecnico di Milano, Milan, Italy 
and reliability in the world. It attracts every year a large number of academics and industry participants, who exchange on both theoretical and practical subjects of interest to various industries.

The articles included in this issue have been selected for scientific quality and to embrace a mix of learning from past decision-making contexts to improve future safety and risk management, as well as modelling issues to provide better tools intended to support the decision-making process.

Grunnan and Fridheim examine the planning and conduct of crisis management exercises to inform useful learning and knowledge sharing for decision-makers. This research is motivated by the role such exercises can play in helping organizations strengthen crisis management capability, identifying possible improvements in planning and developing necessary people skills. Drawing upon practical experiences from a selection of thought and functional exercises, involving a variety of military and civilian actors, Grunnan and Fridheim argue that it is possible to improve the planning and conduct of many exercises, leading to more relevant results and greater benefits for the participants. Problem structuring methods are used to surface and analyze learning points, resulting in a set of success criteria for crisis management exercises with examples of what works well and what does not. The insights shared should be useful for anyone involved in crisis management exercises: from strategic planners to practitioners.

Lubashevskiy, Suzuki, Kanno and Furuta propose a method for making optimal decisions to support recovery management. Motivated by the need for urban sociotechnical systems, such as large cities, to recover after a large-scale disaster event, this article develops a means of determining tactics for managing recovery under major information uncertainties. A socio-technical system is framed in terms of three interdependent elements: a physical connectivity system, citizens' daily life demand, and service systems where the functioning of the latter two depend on the first element. The method proposed aims to deal with the particular challenges of recovery given unreliable information upon which decisions are to be made and the dynamic nature of the decision-making context. For example, in a disaster environment there are many reasons why data collection might comprise impacting the state of knowledge about the true system state. Additionally, possible cascading infrastructure failures means there is a need to reassess damage and react accordingly in making decisions. Lubashevskiy et al. have developed a method based upon step-wise logic and share findings from numerical simulations which allow the applicability of the approach to recovery management decision-making situations to be evaluated.

Haugen and Edwin examine how risk assessment might be usefully conducted during the operation of offshore oil and gas installations. This is an important problem for established off-shore activities, such as the oil and gas sector, and an emerging challenge for newer sectors, such as off-shore wind farms and other renewable energy systems. Haugen and Edwin explore learnings from the oil and gas industry which indicate some common reasons for the relative lack of use of risk assessment to support operational, as opposed to design, decision-making. They find that risk assessment is considered very complicated, having been aimed at risk analysts and not operations personnel on offshore installations, and that the 
assessments could not be updated sufficiently often to reflect changes in risk on a day-by-day basis. Scientifically, Haugen and Edwin examine this problem by investigating the decision context and identifying the distinctive elements of risk assessment between system operation and design. From this deeper appreciation of the characteristics of risk assessment during operation of oil and gas installations, a revised methodology for developing quantitative risk assessments is proposed and tested on an offshore installation, to investigate its feasibility in practice.

Dong, Vinnem and Utne are also interested in decision processes in the off-shore sector, but their focus is upon improving the safety of dynamic positioning operations. Motivated by the fact that dynamic positioning vessels are already widely used for different types of complex marine operations, and this use is increasing, this article examines potential safety improvements grounded in the learning of past incidents. The need to ensure safety for personnel and installations involved is critical and so it is timely to reflect on new risk reduction measures. Drawing upon analysis for the case of loading operations with a dynamic positioning shuttle tanker in offloading from floating production storage and offloading vessels on the Norwegian Continental Shelf, this article highlights insights that might be relevant more generally. The case method adopted is based on three approaches: structured analysis of events and causal relationships; change analysis exploring how observed events deviated from common practice; and barrier analysis identifying technological and administrative barriers which have failed or are missing. Analysis finds that the majority of the accidents are caused by combined technical, human and organizational failures. Based on their study, Dong et al. propose, amongst others, a means of assessing aspects of the actual system to identify potential safety improvements and the development of an on-line risk monitoring and decision support system.

Sørskår and Abrahamsen report research motivated by the need to make decisions under uncertainty in industries regulated for health, safety and the environment. The assume the perspective of the decision-maker tasked with evaluating regulatory intervention options using a form of cost-benefit analysis. Through a critical evaluation of a methodology proposed to the Norwegian government for regulatory evaluation in the petroleum industry, Sørskår and Abrahamsen question the manner in which uncertainty is modelled. They examine different scenarios to explore how much weight should be given to the so-called cautionary principle when making decisions under uncertainty within a regulated industry. A new methodology is proposed which is grounded in the argument that decision-makers should be allowed to adopt a more dynamic approach, where the chosen cost-benefit method better aligns with the context. An example of using this new methodology is presented as an illustration of its proof-of-concept.

Making decisions is fundamental to designing, operating and decommissioning engineering systems safely and reliably. To date, emphasis has often been given to modelling reliability, risk and safety, implying that consideration of the technical method to do this dominates examination of the process of making the end decisions. By the set of selected articles that give specific consideration of the process of making decisions within the reliability, risk and safety context, this Feature Issue provides a distinctive perspective on our application domain. 
In closing this editorial, we thank deeply the authors, the reviewers, the Editor-inChief and all colleagues at the Publishing House, who have contributed to the realization of this special issue.

Lesley, Tim, Matthew and Enrico

Glasgow, Milan and Paris

September 2017 